\title{
SYSTEMIC ANOMALIES IN 77 PATIENTS WITH CONGENITAL ANOPHTHALMOS OR MICROPHTHALMOS
}

\author{
SUSAN TUCKER ${ }^{1 *}$, BARRY JONES $^{2}$ and RICHARD COLLIN ${ }^{1}$ \\ London
}

\begin{abstract}
SUMMARY
Congenital anophthalmos and microphthalmos are rare conditions which can have associated pathology in the second eye and/or systemic anomalies. A retrospective review of 77 patients with congenital anophthalmos or microphthalmos seen at Moorfields Eye Hospital over a 13 year period was performed. A detailed description of the ocular and systemic anomalies present in our series of patients is given, and the current understanding of the pathogenesis of congenital anophthalmos and microphthalmos is reviewed.
\end{abstract}

Congenital anophthalmos and microphthalmos are rare conditions, with prevalence rates for anophthalmos between 0.3 and 0.6 per 10000 births $^{1,2}$ and prevalence rates of $1.4,1.8$ and 3.5 per 10000 births for microphthalmos reported in several large studies. ${ }^{1,3,4}$ Coloboma occurs in 0.7 per 10000 births. ${ }^{1,3}$ It is important to examine these infants for associated ocular and systemic anomalies. ${ }^{1-7}$ The goal of this study was to describe the associated anomalies in our large series of 29 patients with congenital anophthalmos and 48 infants with congenital microphthalmos.

\section{MATERIAL AND METHODS}

The medical records of 77 patients with congenital anophthalmos or microphthalmos seen at Moorfields Eye Hospital between January 1980 and October 1993 were reviewed. On each of these patient's initial hospital visit, a complete history, genetic pedigree and careful physical examination was performed by a paediatrician (B.J.), and then the patient underwent a thorough ophthalmological examination. For the

From: Departments of ${ }^{1}$ Oculoplastic \& Reconstructive Surgery and ${ }^{2}$ Paediatrics, Moorfields Eye Hospital, London, UK. *Current affiliation: Southern Illinois University School of Medicine, USA.

Correspondence to: J. R. O. Collin, MA, FRCS, DO, Moorfields Eye Hospital, City Road, London EC1V 2PD, UK. patients with systemic abnormalities diagnosed or investigated elsewhere, hospital records were obtained from these institutions and reviewed. Patients were categorised as having clinically anophthalmic sockets when there was no evidence of a globe or tissue remnant present. Microphthalmos was diagnosed when axial length, adjusted for age, was below the 5th percentile..$^{89}$ The microphthalmic sockets were further divided into those where only an apical 'remnant' remained; microphthalmic globes with no associated ocular coloboma; and microphthalmic globes associated with ocular coloboma. Karyotype analysis was selectively performed in patients with multiple system disorders, or positive family histories for ocular or systemic disease.

\section{RESULTS}

Table I summarises the demographics of our patients with congenital unilateral anophthalmos (CUA), congenital bilateral anophthalmos (CBA), congenital unilateral microphthalmos (CUM) and congenital bilateral microphthalmos (CBM).

One of the 14 patients with CUA $(7 \%)$ had a positive family history: a male (with one older normal brother) born with CUA, spina bifida and neurogenic bladder whose maternal grandfather had isolated CUA. Another of the 14 patients with CUA (7\%) had a positive systemic family history: a female with CUA, congenital rubella syndrome, microcephaly, ipsilateral brain cyst and alopecia had a brother with Hurler's syndrome.

Two of the 15 patients with CBA (13\%) had positive family histories: a male (only child) with isolated CBA with a maternal aunt born with congenital anophthalmos of one eye and microphthalmos of the other eye; and a male (with two normal brothers) with CBA, pyloric stenosis, mental retardation, left external ear abnormality and normal karyotype, with a positive family history (a maternal 
Table I. Demographics of patients with congenital anophthalmos and microphthalmos

\begin{tabular}{lccccc}
\hline & No. of & Male/female & $\begin{array}{c}\text { Right/left } \\
\text { eye }\end{array}$ & $\begin{array}{c}\text { Family history } \\
\text { of congenital } \\
\text { anophthalmos/ } \\
\text { microphthalmos }\end{array}$ & $\begin{array}{c}\text { Family history } \\
\text { of systemic } \\
\text { anomalies }\end{array}$ \\
\hline Unilateral anophthalmos & 14 & $9 / 5$ & $10 / 4$ & 1 & 1 \\
Bilateral anophthalmos & 15 & $7 / 8$ & - & 2 & 0 \\
Unilateral microphthalmos & 38 & $13 / 25$ & $18 / 20$ & 1 & 3 \\
Bilateral microphthalmos & 10 & $5 / 5$ & - & 0 & 0 \\
\hline
\end{tabular}

aunt with CBA and mental retardation; a maternal male cousin with right-sided CUM; and a maternal great uncle with congenital right-sided CUM. The mothers of all afflicted patients were normal).

One of the 38 patients with CUM (3\%) had a positive family history: a female with CUM with ipsilateral colobomatous iris and disc with a paternal male second cousin with CUM and congenital heart defect, who died at 10 years of age. Three female patients with isolated non-colobomatous CUM had positive family histories of systemic anomalies: (1) a brother with spina bifida and a normal sister; (2) a sister with muscular dystrophy; and (3) a deaf paternal aunt with another paternal aunt born with cleft lip and palate (two normal paternal uncles).

None of our 10 patients with CBM had positive family histories for ocular or systemic disease.

Tables II and III summarise the associated ocular and systemic anomalies in patients with congenital anophthalmos and microphthalmos. The microphthalmos group is further subdivided into those with a tissue remnant only, non-colobomatous microphthalmos and colobomatous microphthalmos.

In our CUA group, 29\% (4/14) had an isolated defect and $21 \%(3 / 14)$ had pathology in the second eye but were otherwise normal. The pathology in the second eye of each of these 3 patients was disc coloboma and retinal detachment, iris coloboma and retinal detachment, and microphthalmos respectively.

In our CBA group, 53\% had an isolated defect. Half our patients with CUA or CBA had associated systemic anomalies, as listed in Table II.

In our CUM 'remnant only' group, 33\% (3/9) had an isolated defect and $11 \%$ (1/9) had congenital nystagmus affecting the fellow eye but were otherwise normal. In our 'non-colobomatous' CUM group $70 \%(14 / 20)$ had an isolated defect, and none had pathology in the second eye. In our 'colobomatous' CUM group 44\% (4/9) had an isolated defect (colobomatous cyst, choroidal coloboma, eyelid coloboma, and choroidal and iris coloboma (2 patients) and 44\% (4/9) had pathology in the second eye but were otherwise normal. Pathology in the second eye consisted of iris coloboma with cataract, bilateral iris and choroidal coloboma ( 2 patients) and disc coloboma.

In our CBM 'remnant only' group, 40\% (2/5) were isolated to the eye and our 3 non-colobomatous CBM and two colobomatous CBM (both with orbital cysts) were isolated. Just over half of patients with CUM or CBM 'remnant only' microphthalmos had

Table II. Associated ocular and systemic pathology in patients with congenital anophthalmos

\begin{tabular}{|c|c|c|}
\hline & Unilateral anophthalmos & Bilateral anophthalmos \\
\hline $\begin{array}{l}\text { Isolated: } \\
\text { Pathology in } 2 \text { nd eye (otherwise normal): } \\
\text { Associated systemic anomalies: }\end{array}$ & $\begin{array}{l}4(29 \%) \\
3(21 \%) \\
7(50 \%) \\
\text { - Deaf, facial defects, webbed hands, } \\
\text { hermaphrodite, colobomatous lids } \\
\text { bilaterally } \\
\text { - Preauricular tags, hemifacial } \\
\text { microsomia, hydrocephalus } \\
\text { - Congenital rubella, microcephaly, } \\
\text { ipsilateral alopecia and brain cyst } \\
\text { - Seizures, 2nd eye staphyloma and } \\
\text { optic nerve dysplasia } \\
\text { - Cleft lip and palate, rudiment ipsilateral } \\
\text { ear, decreased hearing contralateral } \\
\text { ear, cavernous haemangioma occipital } \\
\text { scalp, one hypoplastic kidney } \\
\text { - Ipsilateral facial hemiatrophy, } \\
\text { preauricular tag, large occipital skull } \\
\text { defect, contralateral preauricular tag } \\
\text { and anaesthetic cornea } \\
\text { - Spina bifida, neurogenic bladder } \\
\text { 14 }\end{array}$ & $\begin{array}{l}8(53 \%) \\
\overline{7} \text { ( }(47 \%) \\
\text { - Pyloric stenosis, mental retardation, left ear } \\
\text { external abnormality } \\
\text { - One kidney dyplastic; one rib missing, } \\
\text { thumbs absent } \\
\text { - Bilateral renal dysplasia with renal failure } \\
\text { and urethral hypoplasia } \\
\text { - Corpus collosum dysgenesis } \\
\text { - Hydrocephalus, epilepsy } \\
\text { - Tracheoesophageal atresia } \\
\text { - Right foot 2nd and 3rd toe partial } \\
\text { syndactyly }\end{array}$ \\
\hline
\end{tabular}

\footnotetext{
andividual with family history of congenital anophthalmos or microphthalmos.
}

${ }^{\mathrm{b}}$ Individual with a family history of systemic disease. 
Table III. Associated ocular and systemic pathology in patients with congenital microphthalmos

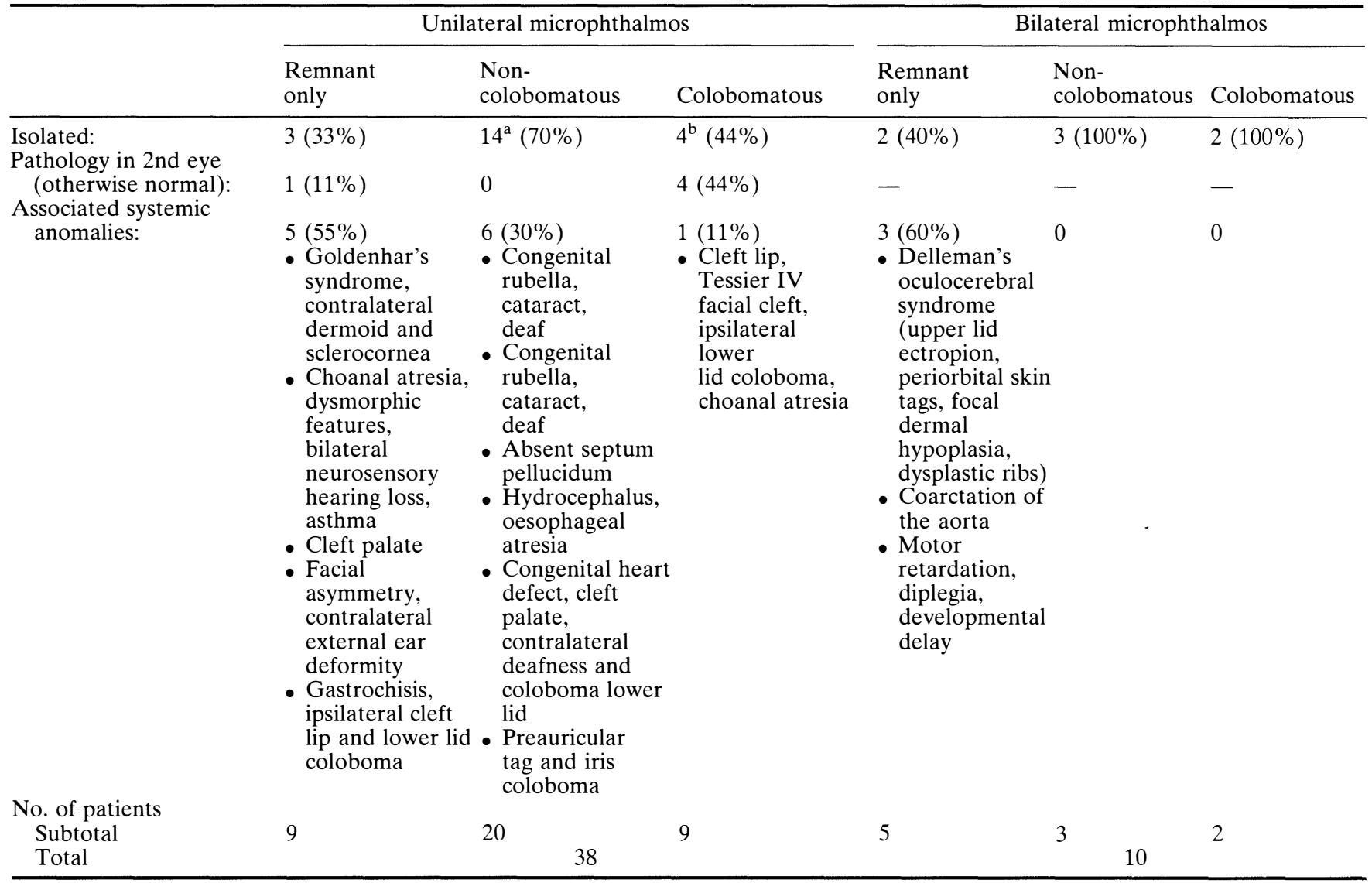

Individual with a family history of congenital anophthalmos or microphthalmos.

${ }^{\mathrm{b}}$ Three individuals with family histories of systemic disease.

associated systemic anomalies. However, fewer of our CUM patients in the remaining two microphthalmic groups had associated systemic anomalies: only 6 of $20(30 \%)$ patients with non-colobomatous CUM; 1 of $9(11 \%)$ with colobomatous CUM, and none of the 3 patients with non-colobomatous CBM, nor the 2 patients with colobomatous CBM.

\section{DISCUSSION}

The congenital anophthalmic-microphthalmic spectrum of disorders are rare entities where uncertainty remains regarding aetiology and the prevalence of systemic anomalies. European registries ${ }^{1,5}$ have reported that between $40 \%$ and $73 \%$ of individuals with either congenital anophthalmos or microphthalmos have associated malformations. In our series of 77 patients we found a $50 \%$ association with systemic anomalies in patients with congenital unilateral or bilateral anophthalmos and congenital unilateral or bilateral 'remnant only' microphthalmos, but lower percentages in our unilateral and bilateral noncolobomatous microphthalmic groups $(30 \%$ and 0 respectively) and our unilateral and bilateral colobomatous microphthalmic groups $(11 \%$ and 0 respectively). Therefore, although histological analysis to confirm the absence of neural ectoderm derivative cells is necessary to distinguish anophthal- mos from 'remnant only' microphthalmos, ${ }^{10,11}$ we found similar rates of associated systemic pathology in both groups. In unilateral cases, pathology was present in the second eye of $21 \%(3 / 14)$ patients with anophthalmos, in $11 \%(1 / 9)$ of patients with 'remnant only' microphthalmos, in none of our 20 patients with 'non-colobomatous' microphthalmos, and in $44 \%(4 / 9)$ of patients with 'colobomatous' microphthalmos. Pathology in the second eye was usually a uveal coloboma, and less commonly coloboma of the disc or eyelid, cataract, or nystagmus. Although Foxman and Cameron's ${ }^{6}$ collective review of several small series suggested major systemic abnormalities in patients with bilateral microphthalmos with cyst, and more minor abnormalities in patients with unilateral microphthalmos with cyst, our 2 patients with bilateral microphthalmos with cyst were otherwise completely normal, as was our 1 patient with unilateral microphthalmos and cyst. However, these differences may well reflect our small number of patient in these groups.

The causes of congenital anophthalmos and microphthalmos are complex and probably multifactorial. ${ }^{12,13}$ We find Warburg's aetiological classification useful: ${ }^{12}$ (1) associations: sporadic with unknown aetiology; (2) genetic: monogenic (autosomal dominant, and less commonly autosomal 
recessive or $\mathrm{X}$-linked recessive) or chromosomal aberrations; and (3) prenatal acquired (teratological agents and intrauterine deformations). Most of our cases of congenitally anophthalmic or microphthalmic sockets were sporadic in occurrence, and therefore fell within the first category, in agreement with the literature. ${ }^{14-17}$

A brief review of embryology is helpful in understanding possible associated anomalies. Embryologically, the organogenic period is between the second and sixth week of gestation. Optic vesicle formation occurs at the fourth week; optic cup and embryonic fissure formation at the fifth week; and closure of the embryonic fissure at the sixth week. ${ }^{18-20}$ Congenital anophthalmos occurs when there is complete failure of budding of the optic vesicle or early arrest of its development with subsequent degeneration. Failure of optic vesicle budding may be 'primary', with involvement of ocular tissue only, or 'secondary', with complete suppression of the forebrain - a lethal condition. Congenital microphthalmos results from incomplete invagination of the optic vesicle into the optic cup or defective closure of the embryonic fissure of the optic vesicle (colobomatous microphthalmos, with uveal coloboma or colobomatous cyst). ${ }^{19-21}$ Disc coloboma occurs from failure of the embryonic fissure to meet the optic stalk, and eyelid coloboma from malunion of surface mesoderm.

During the fourth week of gestation, when the optic vesicle is forming, the branchial apparatus is also starting to develop and subsequently transform into structures of the head and neck. ${ }^{18-20}$ The first branchial arch normally develops into facial tissues; the first cartilage becomes the two middle ear bones (malleus and incus), the first branchial groove becomes the external acoustic meatus; and the nerve of the first arch is the trigeminal nerve. ${ }^{19}$ Various patterns of widespread malformations are a result of associated problems in morphogenesis of the first and second branchial arches occurring embryologically between four and six weeks gestation. Probably the most appropriate name for the wide phenotypic variation of anomalies which result is the 'oculo-auriculo-vertebral spectrum'(OAVS). ${ }^{21}$ Other descriptions used for this spectrum include: 'facio-auriculo-vertebral spectrum, first and second branchial arch syndromes, oculo-auricular vertebral dysplasia, hemifacial microsomia, Goldenhar syndrome, and Goldenhar-Gorlin syndrome'. Many of our patients with congenital unilateral anophthalmos or microphthalmos with associated systemic anomalies fit into this spectrum of disorders.

Because of variations in anatomical expression of the OAVS spectrum of disorders, we elected to list associated abnormal features rather than attempt further categorisation. It is important in any patient born with congenital anophthalmos or microphthal- mos to be aware of and look for these potential systemic associations in the OAVS spectrum of disorders: central nervous system (microcephaly, hydrocephalus, occipital encephalocoele, skull defect, $10 \%$ mental retardation), ${ }^{21,22}$ additional ocular pathology (dermoid, coloboma, Duane's retraction syndrome, decreased corneal sensation (trigeminal nerve abnormality), optic disc hypoplasia) ${ }^{18,23}$ cleft lip, cleft palate; face (hemifacial microsomia, unilateral or bilateral facial hypoplasia, micrognathia); skeletal system (anomalous ribs and limbs); ear (preauricular tags, external and inner ear defects, deafness); heart (ventricular septal defect, patent ductus arteriosus, tetralogy of Fallot, coarctation of the aorta); kidneys; genitourinary system; ${ }^{24}$ and gastrointestinal system. ${ }^{20} \mathrm{~A}$ study of patients with OAVS by Schrander-Sumpel et al. ${ }^{22}$ found a strikingly higher frequency of cerebral anomalies and mental retardation with bilateral ear anomalies, cleft lip/palate and anophthalmia/microphthalmia.

All patients born with congenital anophthalmos or microphthalmos require a thorough clinical examination by a paediatrician looking for associated congenital anomalies of tissues of mesodermal and ectodermal origin, which we found in approximately half our patients with clinical anophthalmos or an ocular remnant only in one or both sockets. We found the incidence of associated systemic pathology in patients with congenital unilateral and bilateral non-colobomatous microphthalmos and unilateral and bilateral colobomatous microphthalmos to be $30 \%, 0,11 \%$ and 0 respectively. In unilateral cases, the second eye should be carefully examined looking for anophthalmos, microphthalmos, coloboma (uveal, disc and eyelid), epibulbar dermoids, corneal opacities, cataracts, retinal detachment and nystagmus. Neuroimaging should be strongly considered for patients with unilateral or bilateral congenital anophthalmos or 'remnant only' microphthalmos, and in non-colobomatous or colobomatous microphthalmic patients where clinical suspicion exists because of other developmental or neurological abnormality.

Key words: Anomalies, Anophthalmos, Congenital, Microphthalmos.

\section{REFERENCES}

1. DeWals P, Lechat MF. Surveillance of congenital anomalies, years 1980-1984. EUROCAT Rep 1987; 2:57-9.

2. Clementi M, Turolla L, Mammi I, Tenconi R. Clinical anophthalmia: an epidemiological study in northeast Italy based on 368256 consecutive births. Teratology 1992;46:551-3.

3. Stoll C, Alembik Y, Dott B, Roth MP. Epidemiology of congenital eye malformations in 131760 consecutive births. Ophthalmic Paediatr Genet 1992;13:179-86.

4. Myrianthopoulos ND. Malformations in children from one to seven years. New York: Alan R Liss, 1985:329. 
5. Gilbert R. 'Cluster' of anophthalmia in Britain. BMJ 1993;307:340-1.

6. Foxman S, Cameron JD. The clinical implications of bilateral microphthalmos with cyst. Am J Pediatr 1984; 97:632-8.

7. Al Frayh AR. Anophthalmia, microcephaly, hypotonia, hypogonadism, failure to thrive and developmental delay. Dysmorph Clin Genet 1987;1:64-6.

8. Harayama K, Amemiya T, Nishimura H. Development of the eyeball during fetal life. J Pediatr Ophthalmol Strabismus 1981;18:37-40.

9. Tucker SM, Enzenauer RW, Levin AV, Morin JD, Hellmann J. Corneal diameter, axial length and intraocular pressure in premature infants. Ophthalmology 1992;99:1296-300.

10. Pearce WG, Nigam S, Rootman J. Primary anophthalmos: histological and genetic features. Can J Ophthalmol 1974;9:141-5.

11. Brunquell PJ, Papale JH, Horton JC, Williams RS, Zgrabik MJ, Albert DM, Hedley-Whyte ET. Sexlinked hereditary bilateral anophthalmos: pathologic and radiologic correlation. Arch Ophthalmol 1984; 102:108-13.

12. Warburg M. Classification of microphthalmos and coloboma. J Med Genet 1993;30:664-9.

13. Zimmerman LE, Font RL. Congenital malformations of the eye: some recent advances in knowledge of the pathogenesis and histopathological characteristics. JAMA 1966;196:684-92.
14. Warburg M. Genetics of microphthalmos. Int Ophthalmol 1981;4:45-65.

15. Goltz RW, Peterson WC, Gorlin RJ, Ravits HG. Focal dermal hypoplasia. Arch Dermatol 1962;86:708-17.

16. Francois J. Hérédité des affections colobomateuses du globe oculaire. Ann Oculist (Paris) 1966;199:545-73.

17. Zeiter HJ. Congenital microphthalmos: a pedigree of four affected siblings and an additional report of fiftyfour sporadic cases. Am J Ophthalmol 1963;55:910-22.

18. Poswillo D. The pathogenesis of the first and second branchial arch syndrome. Oral Surg 1973;35:302-29.

19. Moore KL. Study guide and review manual of human embryology. Philadelphia: WB Saunders, 1975:114.

20. Moore KL. The developing human: clinically oriented embryology, 4th ed. Philadelphia: WB Saunders, 1988: 419.

21. Gorlin RJ, Pindborg JJ, Cohen MM. Syndromes of the head and neck, 3rd ed. New York: McGraw-Hill, 1989: 641.

22. Schrander-Stumpel CTRM, de Die-Smulders CEM, Hennekam RCM, Fryns JP, Bouckaert PXM, Brouwer OF, da Costa JJ, Lommen EJP, Maaswinkel-Mooy PD. Oculoauriculovertebral spectrum and cerebral anomalies. J Med Genet 1992;29:326-31.

23. Budden SS, Robinson GC. Oculoauricular vertebral dysplasia. Am J Dis Child 1973;125:431-3.

24. Ritchey ML, Norbeck J, Huang C, Keating MA, Bloom DA. Urologic manifestations of Goldenhar syndrome. Urology 1994;43:88-91. 